\title{
Spin-aligned RI beams via two-step fragmentation reactions
}

\author{
Y. Ichikawa ${ }^{\mathrm{a}, *}$, H. Ueno $^{\mathrm{a}}$, Y. Ishii ${ }^{\mathrm{b}}$, T. Furukawa ${ }^{\mathrm{c}}$, A. Yoshimi ${ }^{\mathrm{d}}$, D. Kameda ${ }^{\mathrm{a}}$, H. Watanabe ${ }^{\mathrm{a}}$, N. Aoi $^{\mathrm{a}}$, \\ K. Asahi ${ }^{\text {b }}$, D.L. Balabanski ${ }^{\text {e, R. Chevrier }}{ }^{\text {f }}$, J.M. Daugas ${ }^{\text {f }}$, N. Fukuda ${ }^{\text {a }}$, G. Georgiev ${ }^{\text {g }}$, H. Hayashi ${ }^{\mathrm{b}}$, H. Iijima ${ }^{\mathrm{b}}$,

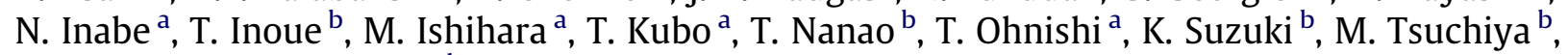 \\ H. Takeda ${ }^{\text {a }}$, M.M. Rajabali ${ }^{\mathrm{h}}$
}

\author{
a RIKEN Nishina Center, RIKEN, 2-1 Hirosawa, Wako, Saitama 351-0198, Japan \\ ${ }^{\mathrm{b}}$ Department of Physics, Tokyo Institute of Technology, 2-12-1 Oh-okayama, Meguro, Tokyo 152-8551, Japan \\ ${ }^{\mathrm{c}}$ Department of Physics, Tokyo Metropolitan University, 1-1 Minami-Osawa, Hachioji, Tokyo 192-0397, Japan \\ ${ }^{\mathrm{d}}$ Research Core for Extreme Quantum World, Okayama University, 3-1-1 Tsushimanaka, Kita, Okayama 700-8530, Japan \\ e Institute for Nuclear Research and Nuclear Energy, Bulgarian Academy of Sciences, BG-1784 Sofia, Bulgaria \\ ${ }^{\mathrm{f}}$ CEA, DAM, DIF, F-91297 Arpajon, France \\ ${ }^{g}$ CSNSM, IN2P3-CNRS, Université Paris-sud, F-91404 Orsay, France \\ ${ }^{\mathrm{h}}$ Instituut voor Kern-en Stralingsfysica, K.U. Leuven, Celestijnenlaan 200D, B-3001 Leuven, Belgium
}

\section{A R T I C L E I N F O}

\section{Article history:}

Received 19 March 2013

Accepted 30 April 2013

Available online 19 June 2013

\section{Keywords:}

Nuclear spin

Nuclear moment

RI beam

\begin{abstract}
A B S T R A C T
A method to produce spin-aligned rare isotope (RI) beams has been developed, where a scheme of twostep projectile fragmentation to produce the RI of interest with high spin alignment and a technique of momentum-dispersion matching are combined. Effectiveness of the present method was demonstrated in an experiment at the RIKEN RIBF, where an RI beam of ${ }^{32} \mathrm{Al}$ with spin alignment of $8(1) \%$ was successfully produced from a primary beam of ${ }^{48} \mathrm{Ca}$, via an intermediate RI of ${ }^{33} \mathrm{Al}$. Figure of merit of the present method was found to be more than 50 times greater than that of the conventional method employing single-step projectile fragmentation.
\end{abstract}

() 2013 Elsevier B.V. All rights reserved.

\section{Introduction}

The technique of spin orientation in RI beams has been playing an important role in the study on nuclear structure through the measurement of nuclear electromagnetic moments. The mechanism of spin orientation in RI beams in a projectile fragmentation (PF) reaction was first revealed in 1990 [1]. The fragmentation of a projectile nucleus in high-energy nucleus-nucleus collisions is described remarkably well by a simple model of "participantspectator" [2], that assumes the projectile-like fragment, produced in the PF reaction, to be a mere spectator of the projectile nucleus. As a spectator, the fragment survives frequent nucleon-nucleon interactions, and the other nucleons (participants) are abraded off through the reaction. In the model, the projectile-like fragment acquires an angular momentum, the orientation of which varies as a function of linear momentum of the outgoing fragment. This implies a unique relation between the spin orientation and the direction of the removed momentum, which can be utilized as an obvious means for producing spin-oriented RI beams. One advantage of this method is that the resulting spin orientation depends

\footnotetext{
* Corresponding author. Present address: Department of Physics, Tokyo Institute of Technology, 2-12-1 Oh-okayama, Meguro, Tokyo 152-8551, Japan.

E-mail address: yuichikawa@phys.titech.ac.jp (Y. Ichikawa).
}

neither on chemical nor atomic properties of the RI. However, the method also shows a drawback in the sense that the spin orientation thus produced in the PF reaction tends to be partially or completely attenuated in cases where the RIs of interest need to be produced through the removal of a large number of nucleons from the projectile. Consequently, significant magnitudes of spin orientation have been obtained only in the vicinity of nuclear species for which a high-intensity primary beam is available. To solve this problem, a breakthrough technique, the two-step PF method with the technique of dispersion matching [3], has been proposed, in which the PF reaction configuration best optimized for high spin alignment can be adopted irrespective of the mass difference between a projectile and the final fragment.

\section{Principle}

In the proposed method, an RI of interest $\left(A_{\mathrm{F}}\right)$ is produced via the one-nucleon removal in the second PF reaction from a nucleus $\left(A_{\mathrm{I}}\right)$ which is an intermediate product of the first PF reaction. In such a scheme magnitude of the spin alignment occurring in the final nucleus $A_{\mathrm{F}}$ is expected to be high by virtue of the simplicity of the reaction [1]. In a simple scheme of this two-step PF, an RI beam of interest is produced after twofold momentum selections by slits, the first of which selects the momentum of the secondary beam 
and the second one the outgoing momentum of the secondary beam. This twofold selection results in tremendous and unnecessary drop in the yield.

An idea of the dispersion matching technique $[4,5]$ is combined to the above two-step PF scheme. The secondary target and the momentum slit are placed at a momentum-dispersive focal plane and a double-achromatic focal plane, respectively. Since the secondary-beam RI is produced within a narrow area on the center of the primary target, its vector, composed of the beam position $x_{0}$ and the momentum deviation from the center $p_{0}$, is expressed as

$\left(\begin{array}{l}x_{0}\left(A_{\mathrm{I}}\right) \\ p_{0}\left(A_{\mathrm{I}}\right)\end{array}\right)=\left(\begin{array}{c}0 \\ \Delta p\end{array}\right)$

where $\Delta p$ is the momentum change generated at the first PF reaction. In the ion optics, the transport matrix $T_{1}$ from an initial focal plane to a momentum-dispersive focal plane through a magnetic separator is expressed as
$T_{1}=\left(\begin{array}{ll}a & b \\ 0 & 1\end{array}\right)$

where $a$ and $b$ denote the magnification through the transportation and the dispersion at the momentum-dispersive focal plane, respectively. The vector on the momentum-dispersive focal plane is written as

$$
\left(\begin{array}{l}
x_{1}\left(A_{\mathrm{I}}\right) \\
p_{1}\left(A_{\mathrm{I}}\right)
\end{array}\right)=T_{1}\left(\begin{array}{l}
x_{0}\left(A_{\mathrm{I}}\right) \\
p_{0}\left(A_{\mathrm{I}}\right)
\end{array}\right)=\left(\begin{array}{c}
b \Delta p \\
\Delta p
\end{array}\right) .
$$

Here the second PF reaction generates the momentum change $\delta p$ in addition to $\Delta p$ as

$$
\left(\begin{array}{l}
x_{1}\left(A_{\mathrm{F}}\right) \\
p_{1}\left(A_{\mathrm{F}}\right)
\end{array}\right)=\left(\begin{array}{c}
b \Delta p \\
\Delta p+\delta p
\end{array}\right) .
$$

The RI of interest $A_{\mathrm{F}}$ is transported to a double-achromatic focal plane with a transportation matrix $T_{2}$,

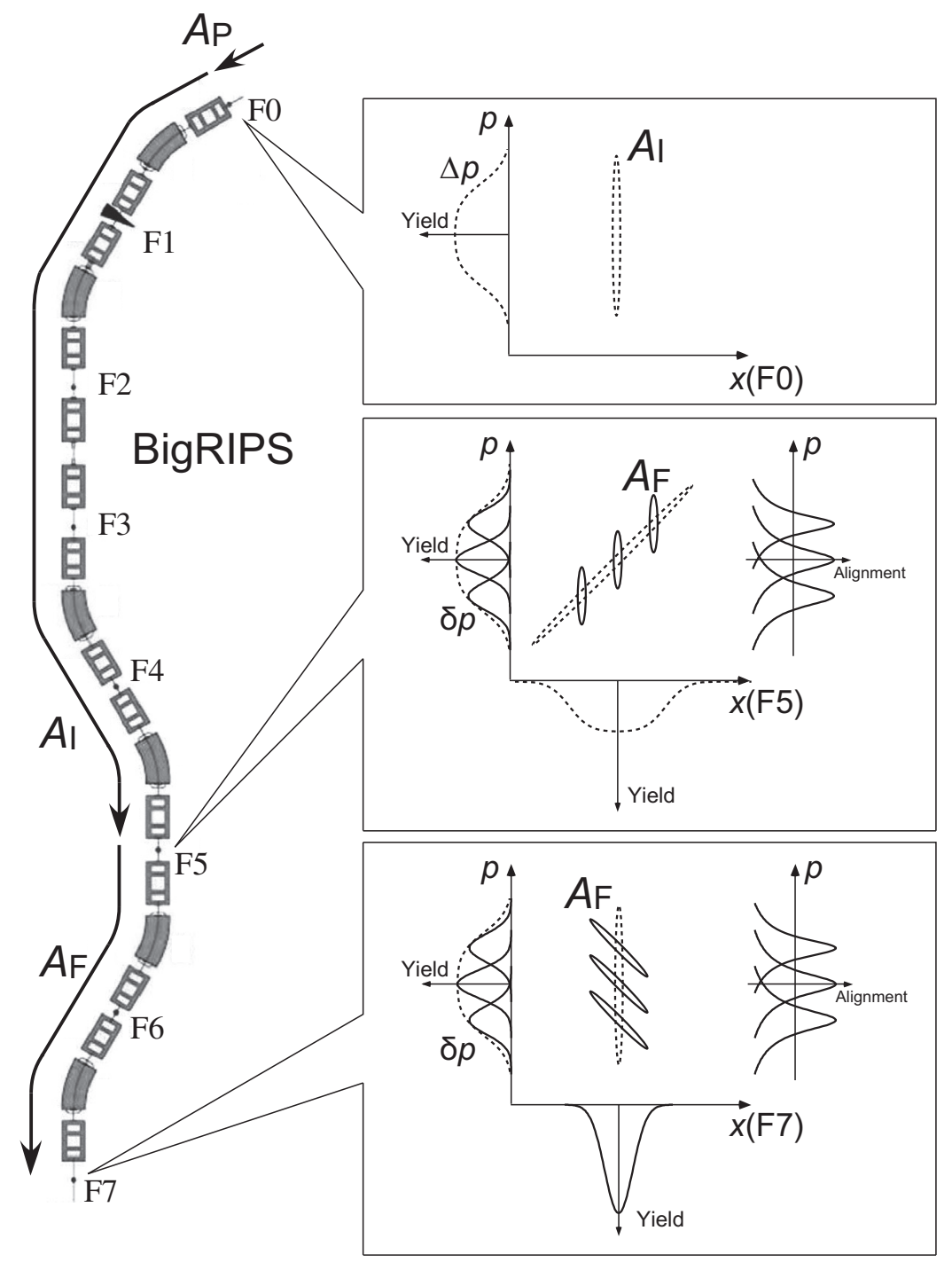

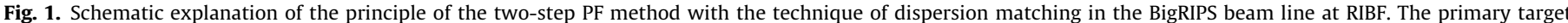

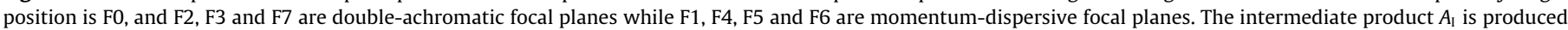

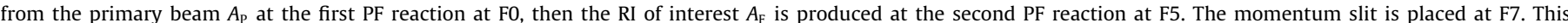

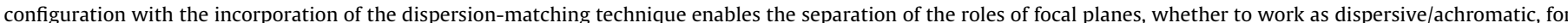

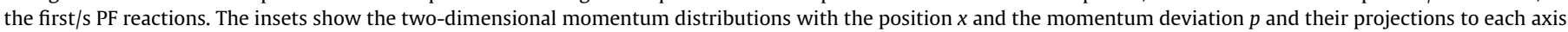

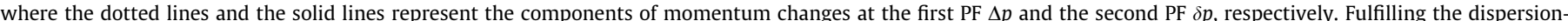

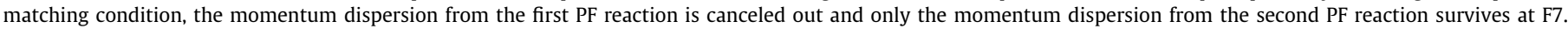
Consequently, a direct extraction of the relative momentum change at the second PF reaction $\delta p$ is realized by a single slit at F7. 
$T_{2}=\left(\begin{array}{ll}c & d \\ 0 & 1\end{array}\right)$

with magnification $c$ and dispersion $d$. In order to cancel out the momentum dispersion (the double-achromatic condition), the right-upper element of the matrix $T_{2} T_{1}$ should be 0 , thus $d=-b c$ (the dispersion matching). Fulfilling the dispersion matching condition, the vector of the tertiary beam (namely the RI beams of interest) at the double-achromatic focal plane is written as

$$
\left(\begin{array}{c}
x_{2}\left(A_{\mathrm{F}}\right) \\
p_{2}\left(A_{\mathrm{F}}\right)
\end{array}\right)=T_{2}\left(\begin{array}{c}
x_{1}\left(A_{\mathrm{F}}\right) \\
p_{1}\left(A_{\mathrm{F}}\right)
\end{array}\right)=\left(\begin{array}{c}
-b c \delta p \\
\Delta p+\delta p
\end{array}\right),
$$

that is, the reaction products that acquire equal amounts of momentum change upon the second reaction are focused onto a single physical location. It is noted here that the quantity that determines the spin alignment is solely the momentum change $\delta p$ in the fragmentation that produces the RI of interest $A_{\mathrm{F}}$, and the spin alignment is not sensitive to the momentum of the incident nucleus. Thus a direct, unique selection of the momentum change $\delta p$ in the second $\mathrm{PF}$ is realized by means of the dispersion matching technique. By applying this technique to the PF-induced spin alignment, the cancelation between spin alignments of opposite signs usually occurring due to the large momentum spread $\Delta p$ is avoided. Schematic explanation of the present method along the BigRIPS beamline [6] is shown in Fig. 1.

\section{Experimental results}

The validity of the present method was tested with the in-flight RI separator BigRIPS [6] at the RIKEN RIBF [7] facility, where an RI beam of ${ }^{32} \mathrm{Al}$ was produced from a primary beam of ${ }^{48} \mathrm{Ca}$ via ${ }^{33} \mathrm{Al}$. The configuration of focal planes in the BigRIPS beam line is shown in Fig. $1 .{ }^{33} \mathrm{Al}$ was produced by a $\mathrm{PF}$ reaction, at the primary target position F0, using the ${ }^{48} \mathrm{Ca}$ beam with energy of $345 \mathrm{MeV} /$ nucleon on a ${ }^{9} \mathrm{Be}$ target with a thickness of $1.85 \mathrm{~g} / \mathrm{cm}^{2}$. The thickness was chosen to provide a maximum production yield for the secondary ${ }^{33} \mathrm{Al}$ beam. A wedge-shaped aluminum degrader with a mean thickness of $4.05 \mathrm{~g} / \mathrm{cm}^{2}$ was placed at the first momentum-dispersive focal plane $\mathrm{F} 1$, where the momentum acceptance was set to $\pm 3 \%$. The secondary ${ }^{33} \mathrm{Al}$ beam was sent to a secondary wedgeshaped aluminum target with a mean thickness of $2.70 \mathrm{~g} / \mathrm{cm}^{2}$, placed at the second momentum-dispersive focal plane F5. The thickness of the secondary target was chosen such that the energy loss from the target was comparable with the Goldhaber width [8] for the one-nucleon removal. The ${ }^{32} \mathrm{Al}$ nuclei, including those in isomeric state ${ }^{32 \mathrm{~m}} \mathrm{Al}[9,10]$, were produced through a PF reaction involving the removal of one neutron from ${ }^{33} \mathrm{Al}$. The ${ }^{32} \mathrm{Al}$ beam was subsequently transported to the focal plane F7 whereby the momentum dispersion in F5-F7 was tuned to have the same magnitude and opposite sign as that in F0-F5, so as to cancel out the momentum dispersion originating from the first PF reaction. The slit at F7 was used to select a region of momentum change at the second $\mathrm{PF}$ reaction as $\pm 0.15 \%$ about the center of the relative momentum distribution.

The ${ }^{32 m} \mathrm{Al}$ beam was sent to an experimental apparatus placed in a focal plane after F7 for the time-differential perturbed angular distribution (TDPAD) measurement. The TDPAD apparatus consisted of a Cu crystal stopper, a dipole magnet, Ge detectors, a plastic scintillator and a collimator, as shown in Fig. 2. The Cu stopper was $3.0 \mathrm{~mm}$ in thickness and $30 \times 30 \mathrm{~mm}^{2}$ in area, and the dipole magnet provided a static magnetic field $B_{0}=0.259 \mathrm{~T}$ in vertical direction. ${ }^{32 m} \mathrm{Al}$ were implanted into the $\mathrm{Cu}$ crystal, and the deexcitation $\gamma$ rays were detected with four Ge detectors located at a distance of $7.0 \mathrm{~cm}$ from the stopper and at angles of $\pm 45^{\circ}$ and $\pm 135^{\circ}$ with respect to the beam axis. The relative detection effi-

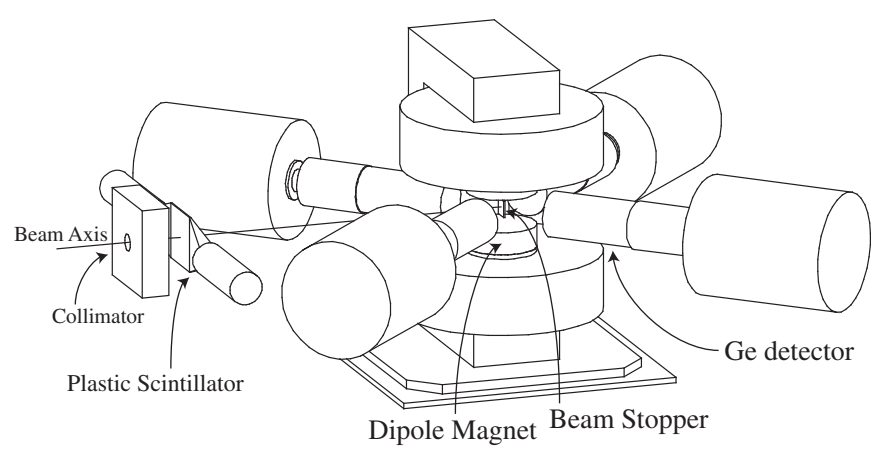

Fig. 2. TDPAD apparatus.

ciency was $35 \%$ for one and $15-20 \%$ for the other three detectors. The plastic scintillator with a thickness of $0.1 \mathrm{~mm}$ was placed upstream of the stopper. Its signal provided the time-zero trigger for the TDPAD measurement. The TDPAD apparatus enabled us to determine the degree of spin alignment in the RI beam of ${ }^{32 m} \mathrm{Al}$ by observing the changes in the anisotropy of the de-excitation $\gamma$ rays, emitted from spin-aligned ${ }^{32 m} \mathrm{Al}$ in synchronization with the spin precession under an external magnetic field.

The degree of spin alignment in ${ }^{32 m} \mathrm{Al}$ was determined from a ratio $R(t)$, defined as

$R(t)=\frac{N_{13}(t)-\epsilon N_{24}(t)}{N_{13}(t)+\epsilon N_{24}(t)}$

where $N_{13}\left(N_{24}\right)$ is the sum of the photo-peak count rates at Ge 1 and Ge 3 (Ge 2 and Ge 4), which are two pairs of Ge detectors placed diagonally to each other, and $\epsilon$ denotes a correction factor for the detection efficiency. Theoretically, the $R(t)$ ratio is expressed as a function of $t$ as

$R(t)=\frac{3 A_{22}}{4+A_{22}} \cos 2\left(\omega_{\mathrm{L}} t+\alpha\right)$,

in terms of the rank-two anisotropy parameter $A_{22}$, which is defined as $A_{22}=A B_{2} F_{2}$. Terms with higher ranks were evaluated to be negligible in the present case of ${ }^{32 m} \mathrm{Al}$. Here, $A$ denotes the degree of spin alignment

$A=\sum_{m} \frac{3 m^{2}-I(I+1)}{I(2 I-1)} a(m)$,

where $a(m)$ is the occupation probability for magnetic sublevel $m$, and $I$ the nuclear spin. $B_{2}$ is the statistical tensor for complete alignment, and $F_{2}$ is the radiation parameter [11]. The parameter $\omega_{\mathrm{L}}$ (Larmor frequency) is given by $\omega_{\mathrm{L}}=g \mu_{\mathrm{N}} B_{0} / h$, where $g$ is the $g$-factor of ${ }^{32 m} \mathrm{Al}$ in units of the nuclear magneton $\mu_{\mathrm{N}}$, and $\alpha$ is the initial phase of $R(t)$.

In the $\gamma$-ray spectrum obtained with the Ge detectors, 222-keV de-excitation $\gamma$ rays, together with $735-\mathrm{keV}$ cascade $\gamma$ rays originating from the isomeric state ${ }^{32 m} \mathrm{Al}$, are observed as peaks. In the deduction of the degree of spin alignment, the 222-keV $\gamma$ rays which directly de-excited the isomeric state were used because the spin alignment would be attenuated through the cascade decays. The time variations $N_{13}(t)$ and $N_{24}(t)$ of the intensities for the 222-keV peak were obtained with detectors pairs Ge 1-3 and Ge $2-4$, respectively, as shown in Fig. 3 (a). The $R(t)$ ratio, evaluated according to Eq. 7, is shown in Fig. 3 (b).

From the least $\chi^{2}$ fitting of the theoretical function of Eq. 8 to the experimental $R(t)$ ratio of Eq. 7, we obtained the degree of spin alignment as $A=8(1) \%$, and the $g$-factor of ${ }^{32 m} \mathrm{Al}$ was determined for the first time to be $g=1.32(1)$. The obtained $R(t)$ plot was fitted by Eq. 8 with a fixed parameter of $\alpha=0$. This is because the beamaxis at F5 is parallel to that at the final focal plane and the preces- 


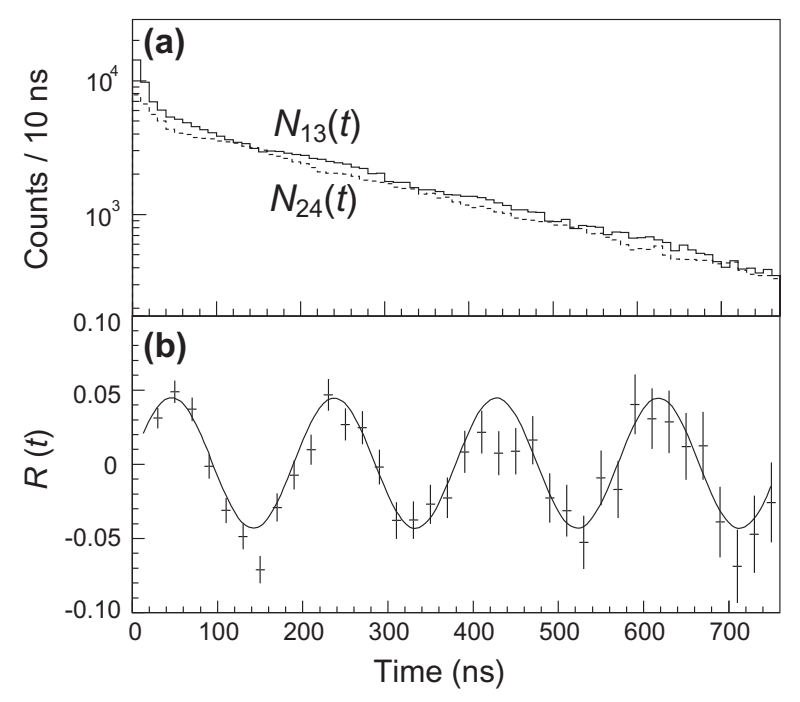

Fig. 3. (a) Time variations of $N_{13}(t)$ (solid line) and $N_{24}(t)$ (dashed line) for 222-keV $\gamma$ rays. Events around $t=0$ originate from prompt $\gamma$ rays. (b) $R(t)$ ratio deduced from $N_{13}(t)$ and $N_{24}(t)$. The solid line represents the $R(t)$ function after fitting to the experimental $R(t)$. In both figures, the horizontal axes represent the time difference of the signals at either of the Ge detector pairs relative to the beam particle signal at a plastic scintillator placed in front of the stopper.

sion through dipole magnets between F5 and F7 is canceled. Also, the spin and parity of the isomeric state were assigned to be $I^{\pi}=4^{+}$through comparison of the $g$-factor with theoretical calculations [12].

In order to check that the dispersion matching was performed well, a remeasurement of the degree of spin alignment was also performed during the experiment, in which the momentum acceptance in the F5 focal plane was narrowed to be $\pm 0.5 \%$, while maintaining other conditions unchanged. This measurement corresponded to the two-step PF reaction without the dispersion matching. The degree of spin alignment derived from this measurement was $9(2) \%$, which is consistent with the above value obtained with the proposed method, $8(1) \%$. Thus, the validity of the dispersion matching to enhance the yield of the RI beam of interest with keeping the magnitude of spin alignment was confirmed.

\section{Discussion}

In order to evaluate the figure of merit compared to that of the conventional method employing single-step PF, a supplementary measurement was also carried out, where ${ }^{32} \mathrm{Al}$ was directly produced from ${ }^{48} \mathrm{Ca}$ by a single-step PF reaction on a $4-\mathrm{mm}$ thick Be target. The thickness was chosen such that the energy loss in the target was comparable with the Goldhaber width [8] for 16-nucleons removal. In this measurement, the degree of spin alignment was less than $0.8 \%$ in $2 \sigma$ confidence level. The yields of ${ }^{32 m} \mathrm{Al}$ at the experimental apparatus were 0.54(5) kcps and 0.87(6) kcps for the two-step PF and the single-step PF measurements, respectively, where intensity of the primary beam at the single-step PF measurement was deliberately attenuated by a factor of $1 / 100$ to avoid saturation in the counting rate at the data acquisition system. The figure of merit (FOM) which was defined as proportional to the yield and the square of the degree of spin alignment, was compared on the basis of actual effectiveness without correction for the attenuation, then the FOM of the present method was found to be more than 50 times greater than that of the conventional method in this case of ${ }^{32} \mathrm{Al}$. The superiority in FOM of the new method over the single-step PF method should be even more pronounced for nuclei located farther from the primary beam.

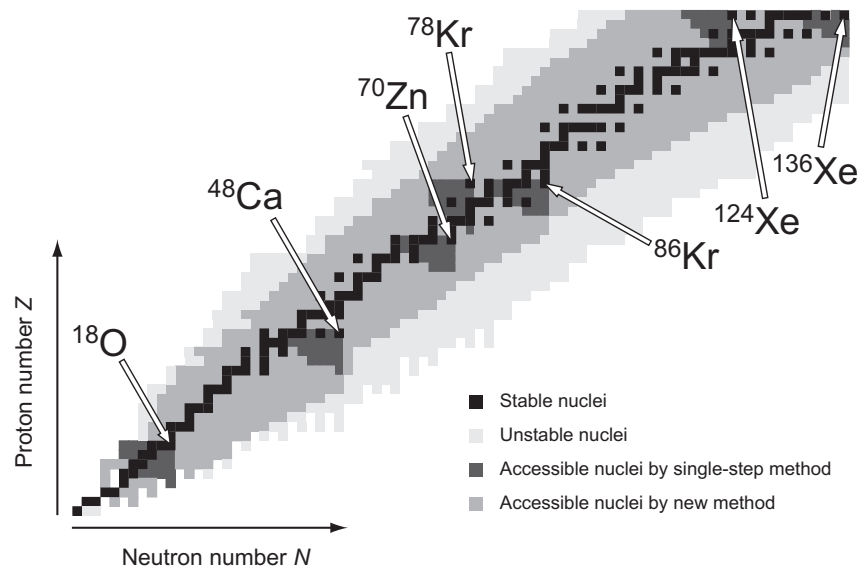

Fig. 4. Nuclear chart indicating "accessible" nuclei. The expression accessible here means that the RI of interest is producible with its spin aligned and with the production yield sufficiently large in order to determine the $g$-factor of its isomeric state with a $5 \sigma$ confidence level in a one-day beam time. The following conditions are assumed: Primary beams are restricted to the typical species available with high intensity at RIBF [7]. The degree of spin alignment is $10 \%$ for the one-nucleon removal from the beam particle, and reduces exponentially to $1 \%$ for the 10 nucleons removal, as is empirically known. The intensities of primary beams actually available at RIBF are assumed. The cross sections for the PF reactions are estimated based on the parameter sets called EPAX2 [13], and cross sections for the secondary PF reaction are assumed to be $1 / 1000$ as typical. Isomeric to ground state population ratio for the RI of interest in the PF reaction is $50 \%$. The external magnetic field for the TDPAD measurement is 1 Tesla.

Fig. 4 shows the result of numerical simulations for the accessibility of unstable nuclei, in terms of spin alignment, via the twostep PF and the single-step PF methods. In this simulation, primary beams are restricted to the typical species which are available with high intensity at RIBF [7]. The present method is expected to enable us to substantially broaden the accessible scope of spinaligned RI beams. In addition to the simplest case that the RI of interest is produced through the one-nucleon removal reaction, the two-step scheme presented in this paper is also applicable to the few-nucleons removal reaction as well as few-nucleon pickup reaction [14].

\section{Summary}

We have proposed a new method to produce spin-aligned RI beams by the two-step PF with a technique of momentum-dispersion matching. Spin alignment of the RI beam of interest is maximized by the two-step PF reactions employing one-nucleon removal, as well as the yield of the RI beam is enhanced by the dispersion matching technique with keeping the maximized spin alignment. The effectiveness of the method was verified in the experiment at the RIKEN RIBF, in which the RI beam of ${ }^{32} \mathrm{Al}$ with a degree of spin alignment of $8(1) \%$ was produced from the primary beam of ${ }^{48} \mathrm{Ca}$ via the intermediate product of ${ }^{33} \mathrm{Al}$. The FOM for the present method was found to be greater than that of the conventional method by a factor of more than 50 . Numerical simulations indicate that the present method substantially expands the region of accessible nuclei in nuclear chart.

\section{Acknowledgements}

Experiments were performed under Program No. NP0702RIBF018 at RIBF, operated by RIKEN Nishina Center and CNS, The University of Tokyo. We thank the RIKEN Ring Cyclotron staff for their cooperation during experiments. Y.I. is grateful to the Special Postdoctoral Researchers Program, RIKEN. This work was partly 
supported by the JSPS KAKENHI (22340071 and 20532089), by the JSPS and MAEE under the Japan-France Integrated Action Program (SAKURA), and by the Bulgarian National Fund (grant DID-02/16).

\section{References}

[1] K. Asahi et al., Phys. Lett. B 251 (1990) 488.

[2] J. Hüfner, M.C. Nemes, Phys. Rev. C 23 (1981) 2538.

[3] Y. Ichikawa et al., Nat. Phys. 8 (2012) 918.

[4] B.L. Cohen et al., Rev. Sci. Instr. 30 (1959) 415
5] H.G. Blosser et al., Nucl. Instr. Meth. 91 (1971) 61.

[6] T. Kubo et al., Nucl. Instr. Meth. B 204 (2003) 97.

[7] Y. Yano et al., Nucl. Instr. Meth. B 261 (2007) 1009.

[8] A.-S. Goldhaber, Phys. Lett. B 53 (1974) 306.

[9] M. Robinson et al., Phys. Rev. C 52 (1996) R1465.

[10] S. Grévy et al., Nucl. Phys. A 734 (2004) 369.

[11] H. Morinaga, T. Yamazaki, In-Beam Gamma-Ray Spectroscopy, North-Holland, Amsterdam, 1976

[12] Y. Ichikawa et al., in preparation.

[13] K. Sümmerer, B. Blank, Phys. Rev. C 61 (2000) 034607.

[14] D.E. Groh et al., Phys. Rev. Lett. 90 (2003) 202502. 